\title{
Discussion of anti-interference detection method based on CW small signal
}

\author{
Zhang ling ${ }^{1, a}$ \\ ${ }^{1}$ No.3 Renmin East Road,Kunming,Yunnan,650051,China. \\ akmjlz@163.com
}

Keywords : electromagnetic signal, anti-interference detection, energy identification

\begin{abstract}
In this paper, four anti-interference detection methods based on a CW small-signal are discussed separately. First the basic principles of various methods about the energy identification, frequency discrimination, SNR identification, pulse width identification are introduced, and then their advantages and disadvantages are analysed. Finally these measures can ensure sensitivity and effectively improve the detection capability of the system. when environment is disturbed by complex electromagnet
\end{abstract}

\section{Introduction}

In a signal detection equipment, target launch is received by equipment receiving sensor, system is requested to real-time detect frequency and phase of the target signal, and to produce the echo of the same frequency and phase in a limited time. The decayse of signal enegy so fastly in disseminating(propagation), in order to increase the range, equipment is needed to improve the detection sensitivity. On the other hand, complex Environmental makes the system receive some random interference, the interference may be brought by underwater environment change, also may be caused by system circuit. The frequency of the jamming signal and real signal is at the same band, but the instantaneous impulse energy of jamming signal is often bigger than the real signal, so voltage general detection methods are usually easy to mistakenly identified to jamming signal. When these inherent disturbance factors cannot be ruled out from the hardware, you must consider from the software detecting means to take measures to improve the anti-interference ability of the system.

At the software, improving the detection sensitivity and anti-jamming is a pair of contradictions. In order to detect the small target signal without interference signal, it is important to analyse of the characteristics of interference signals and real, then to find the difference between the two and take corresponding solutions.

In this article, the interference signal and real signal are simulated firstly according to the actual circumstance, then the similarities and differences of both are analysed. The purpose is to find an effective detection method.

\section{Composition and working principle of the system}

Figure 1 is the system structure diagram. The system is composed by the electric charge sensor, preamplifier circuit, signal conditioning circuit, signal processing circuit, signal processing circuit, power amplifier circuit and transmit sensor, etc. The receiving sensor converts the electromagnetic waves to electrical signals which are amplified, filtered .After amplitude adjustment, signal acquisition, signal analysis, the target signals are identified. Signal processing equipment forms echo data, which is converted to analog signal by the D/A conversion. The analog signal is amplified, filtered, formed to differential drive signals, which are amplified by the power amplifier circuit and are loaded into the transmitter.

Figure 2 is the hardware structure diagram of digital signal processing platform . The system has three channels of $\mathrm{A} / \mathrm{D}$ and $\mathrm{D} / \mathrm{A}$. The corresponding echo is generated by the system according to a certain algorithm. when any one of the A/D channels detects target signals. 


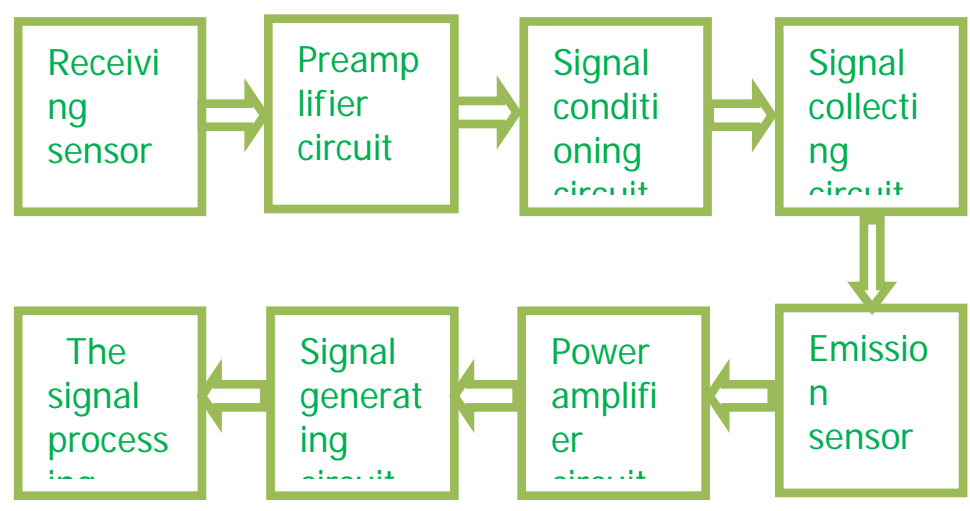

Fig. 1 System block diagram

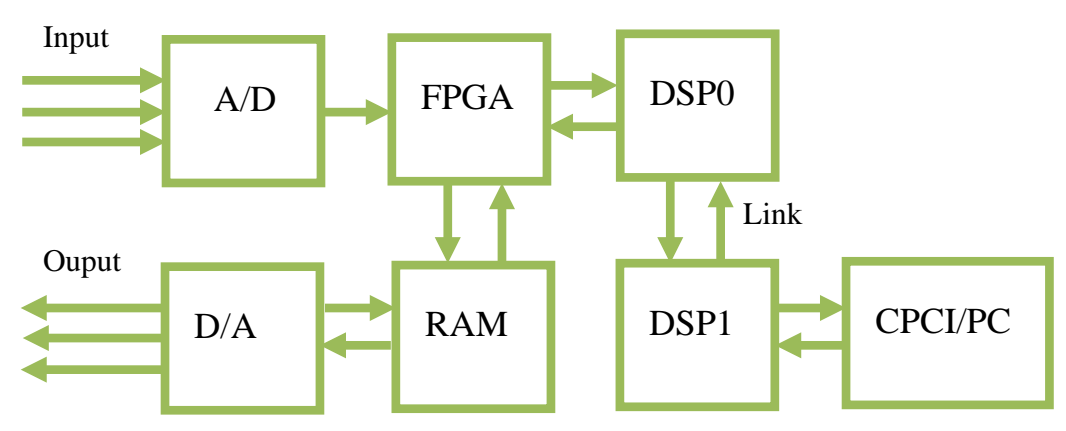

Fig. 2 Hardware platform of Signal Detection System

In the platform , AD converts analog signals into digital signal, which is amplified and filted by FPGA , then is send to DSP0, by which AD code is converted to floating-point format. The floating-point datas are send to DSP1 by the link . DSP1 is responsible for signal analysis and detection and calculates the characteristic including frequency and phase, when target signal is detected, the characteristic is send back to DSP0, which controls FPGA to generate echo.

\section{Anti-interference signal detection method}

\section{Overview on signal detection method}

Firstly, the basic algorithm of signal detection is introduced. The basic characteristics of the signal includes frequency, voltage, pulse width, and signal-to-noise ratio(SNR), etc. Spectrum of digital filter output is calculated to generate the frequency, the energy and SNR . These characteristic values are compared with experience threshold, if signal is effective, continuous time length of effective signal is width. When the pulse width reaches a certain threshold, it can be judged that the target signals comes.

In the stage of system development, in the laboratory ,when simulation signal is used as input, target signals can be stably detect only depending on frequency and voltage. But when the device is applied to the actual environment, it is often affected by the other functional units of system, and it will receive a lot of jamming signals. In test it is found that the device will receive the periodic impact signal, its resonance is in-band signals, as shown in figure 3, which has a big impact on the detection, and will influence the equipment normal work if it is not eliminated.

Usually, the real signal is identified by judging the voltage and frequency . the characteristics of frequency and voltage is relatively stable, but the anti-interference ability is low, jamming signals 
often have the same frequency and voltage as real signals. Relatively, the SNR is more sensitive, which can distinguish between the real signal and interference signal, but sometimes is unstable, judging SNR alone can cause negatives of real signals.

The target signal can also be identified by pulse width, usually the width of the jamming signal is lesser, but there is a lack of real-time performance in the method of pulse width. It is need to improve the detection sensitivity and eliminate random interference, by developing the advantage of each characteristic and combining effectively various methods, but it become the difficulty of the engineering application. This article will look for solutions in simulation,.

Response speed should be taken into account for real-time system. Time domain calculation is more time-consuming than frequency domain, so time domain calculation is likely too late to response for large amount of calculation system. For small amount of calculation system, a choice can be made by weighing the speed and accuracy of the two. Both time domain and frequency domain, the calculation precision of frequency and time consuming is proportional , the difference is that time domain calculation needs high sampling rate, the frequency domain calculation dose not need.

\section{Signal-to-noise ratio method}

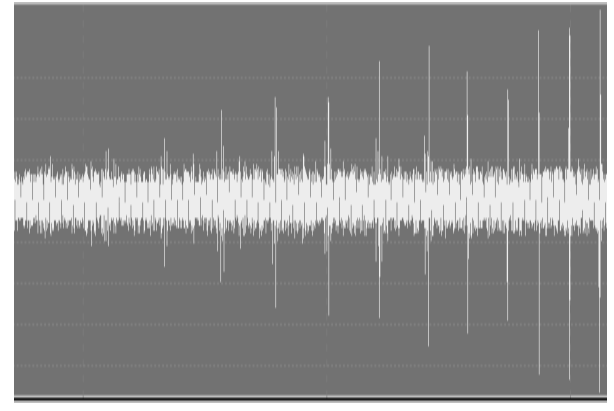

Fig. 3 Periodic interference signal

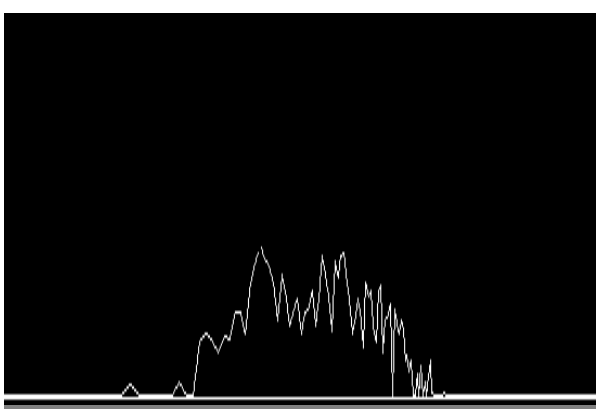

Fig. 4 Power spectrum simulation of jamming

Equipment needs to test for a signal from the target, assuming that frequency band of the target signal is from the fa to fb, start-stop frequency of system filter is from fa - fc to $\mathrm{fb}+\mathrm{fc}$. System receives the signals which are filtered into narrow-band. In order to improve the detection sensitivity, namely tiny target signal is detected as far as possible. The voltage threshold is usually very low, only slightly higher than noise. System offen receives periodic disturbance signal, voltage of which is higher than system Settings detection threshold amplitude, instantaneous voltage spike is filtered into in-band signal pulse with certain width.

Usually in order to cover all the target frequency bandwidth, when the voltage is calculated ,the integral range would be set from fa to fb. Then the noise signal voltage integral is as large as a weak real signal voltage integral, resulting in false check. Figures 4 and 5, respectively is the power spectrum simulation of jamming signal and real signal line, the former energy is uniformly distributed on the fa to fb range, the latter energy is concentrated.

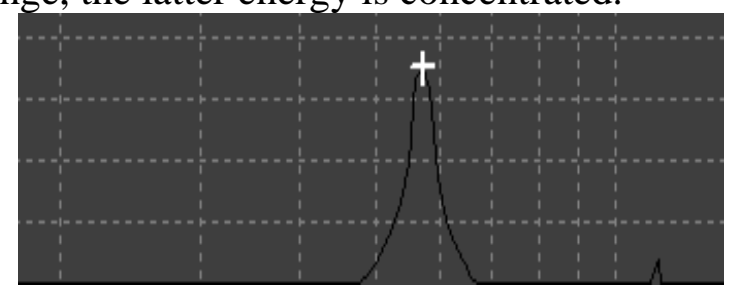

Fig. 5 Tiny, signal line simulation scope of energy

Two signal lines within the scope of the fa to fb integral value quite, only checking by voltage is likely to cause mistake. The following will judge the signal-to-noise ratios to eliminate interference signals. The difference between the two is that spectrum lines of small signal is sharper than and jamming signal, namely energy concentration. In order to reflect the characteristics, the calculation of signal-to-noise ratio, needs to narrow the molecular integral range. The smaller molecular 
integral range ,the sharper degree of the response spectrum, which can distinguish jamming signal. Molecular integral center can be looked at according to the spectral peak, near the point to determine a range.
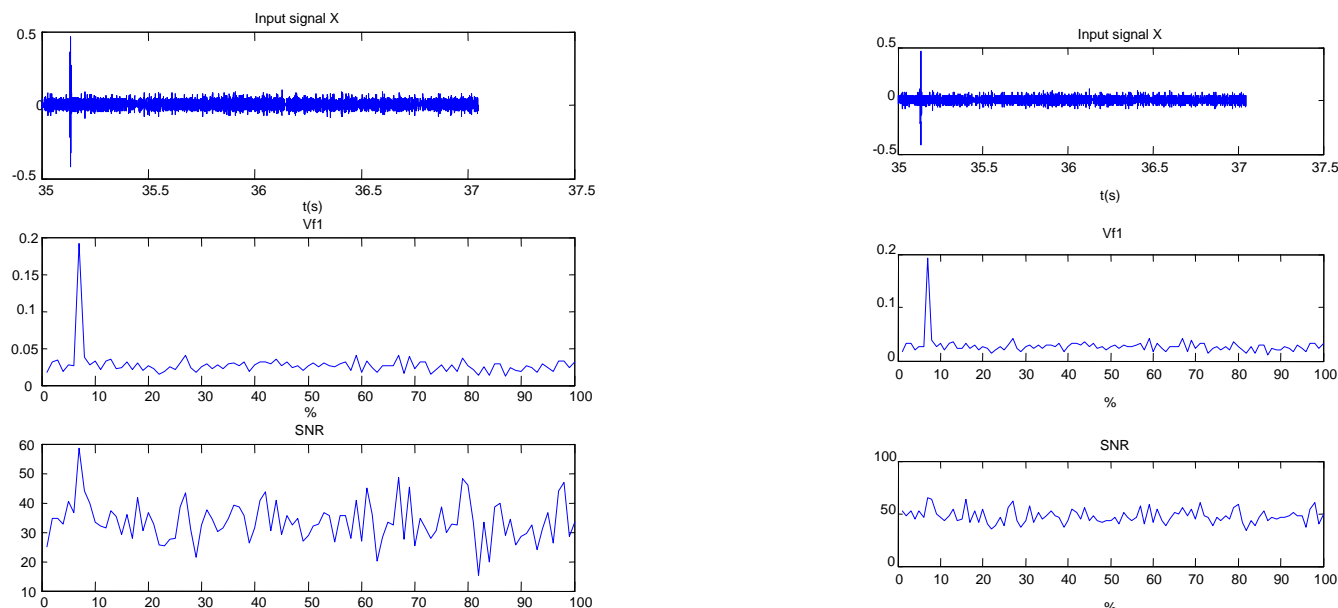

Fig. 6 Molecular score 10 points, the voltage and the signal-to-noise ratio can eliminate interference.

Fig. 7 Molecular integral three points, the signal-to-noise ratio can be ruled out
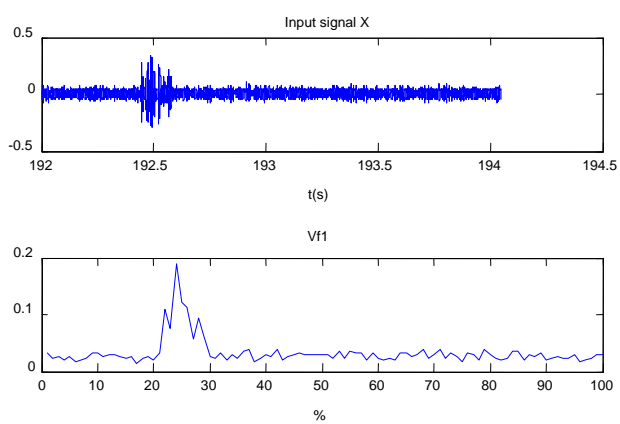

SNR

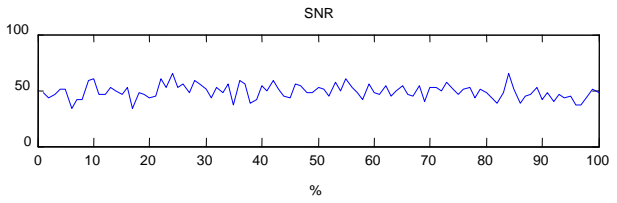

Fig. 8 Molecular integral three points, the signal-to-noise ratio can be ruled out

As shown in figure 6, 7, 8, each figure composed by three parts, the above is the interference signal of time domain graph, the middle is voltage figure, the below is the signal-to-noise ratio figure. Figure 6 and figure 7 are energy calculated by different integral range on the same narrow jamming signal. Small molecular integral when calculating SNR range, can eliminate interference. Figure 8 is wide interference, small molecular integral when calculating SNR is equally effective.

Above analysis is based on simulation connected with practical application. The different systems and different application environment has its own characteristics, targeted analysis should be taken.

\section{Pulse width method}

Interference signals and small signal, another difference is that interference signal in time domain is narrow, the real signal is relatively wide. According to the sampling clock signal is divided into segments, when the first detected incident signal frequency and voltage are up to par, 
the second signal is tested continuelly. If the frequency and voltage are up to par ,and frequency is close to the last ,it can be determined as the real signal arriving.

Checking n consecutive segments can lengthen pulse width. No matter how many segments, each frequency must be accurate approximation, ensure that the target signal is CW , or it may be jamming signal. It has the advantage of high reliability to eliminate interference by pulse width, the downside is time-consuming. It needs to accurately measure to ensure the response time for real-time response system. SNR, by contrast ,only needs a signal sampling time, spend little time, strong real-time performance.

\section{The intermittent transceiver method}

For system which generates the real-time echo, in order to prevent the system from interfering signal or characteristic value detected error, which cause a false signal response. The intermittent test strategy can be adopted. The upper software sets the testing cycle, the system the forbidden time, which is to prevent the launch signal of system itself been received back, forbidden time is slightly longer than signal recognition time.

\section{Conclusion}

Based on a electromagnetic signal detection system as an example, this paper introduces the structure and principle of system, hardware platform, and further expounds disturbance in the detecting process of practical application situation. And the disturbance signal is analysed by algorithm, the results are compared. The method applied to the projects has achieved good effect. This paper, mainly introduces a method of anti-jamming analysis ,different system due to different application environment, the interference will be different. The key is, analyzing the specific issues to find a reasonable solution.

\section{References}

[1] Digital signal processing theory and algorithms, and implementation, tsinghua university press.

[2] Digital signal processing and matlab, the electronic industrial press.

[3] Liu shuming. Su tao. Luo Junhui .TigerSHARC DSP application system design.

[4] He Ke, Zhang Xiaomin, application of ultra short baseline array in underwater target detection and location of the master's thesis, Northwestern Polytechnic University, 2004.

[5] Yang Yichun, Ma Chi, research, accurate delay algorithm for fast correlation peak refinement acoustic Sinica, 2003.3. 